\title{
Surgical resection of scalp arteriovenous malformation: Case illustration
}

\author{
Sadaharu Tabuchi \\ Department of Neurosurgery, Tottori Prefectural Central Hospital, Tottori, Japan; Corresponding Author: tabuchis@pref.tottori.jp
}

Received 4 March 2013; revised 10 April 2013; accepted 10 May 2013

Copyright (c) 2013 Sadaharu Tabuchi. This is an open access article distributed under the Creative Commons Attribution License, which permits unrestricted use, distribution, and reproduction in any medium, provided the original work is properly cited.

\begin{abstract}
Arteriovenous malformation (AVM) of the scalp is a rare lesion whose natural history remains to be elucidated. The report describes a case of a patient with a history of minor head trauma who presented with spontaneous bleeding from the scalp due to a relatively small scalp AVM. Several imaging modalities were used to establish the diagnosis, map the vascular malformation, and plan management. The optimal treatment strategy for this type of lesion remains controversial. Based on the size of AVM and the number of feeding arteries, this patient was treated with complete surgical excision alone. The clinical course was good, without postoperative complication or recurrence. Three-dimensional computed tomography angiography (3D-CTA) was useful for diagnosis, management and follow-up of this small extracranial scalp AVM.
\end{abstract}

Keywords: Arteriovenous Malformation; Scalp; Resection; Three-Dimensional Computed

Tomography Angiography

\section{INTRODUCTION}

Arteriovenous malformation (AVM) of the scalp is a rare vascular lesion that is characterized by abnormal fistulous connections between supplying arteries and draining veins without an intervening capillary network in the subcutaneous plane of the scalp [1-4]. This report describes a case of a patient with a scalp AVM who presented with spontaneous bleeding from the lesion. The optimal treatment strategy for this type of lesion remains controversial. This patient underwent surgical excision of the AVM with favorable results.

\section{CASE REPORT}

A 75-year-old male presented with a 3-month history of a pulsatile reddish soft mass in the right frontoparietal region, measuring $5 \mathrm{~cm}$ on its longest axis (Figure 1(a)). He had a history of mild head trauma. He suddenly developed severe bleeding from the scalp lesion and was referred to us for treatment of the scalp mass. Computed tomography (CT) demonstrated a mass that was completely within the scalp and was not associated with bone or periosteum. Three-dimensional CT angiography (3DCTA) suggested the presence of an AVM in the scalp (Figure 1(b)). Selective digital subtraction angiography (DSA) revealed a scalp AVM fed by the superficial temporal artery (STA) and draining into the superficial scalp veins (Figure 1(c)). Surgical total resection under operative microscopy with stepwise dissection and hemostasis was performed without complication. This resulted in successful excision of the AVM, including the feeding artery and draining vein (Figure 1(d)). Intraoperative near-infrared indocyanine green (ICG) videoangiography showed resolution of the AVM. Histopathologic examination of resected tissue confirmed a diagnosis of AVM. The postoperative course was uneventful, with no recurrence at the 2-year follow-up.

\section{DISCUSSION}

AVM of the scalp is a rare lesion. Its management is difficult because of its highly complex vascular anatomy and the possible cosmetic complications of surgery. Clinical signs are dependent on the size of the lesion, but hemorrhage is generally uncommon. The diagnosis is usually made by direct physical observation, because these lesions are quite noticeable in most cases. Several imaging modalities can be used to map the vascular malformation and to help plan management, and angiography is especially useful for diagnosis. Treatment options include surgical excision, ligation of feeding vessels, and transarterial and transvenous embolization [2,4-6]. Historically, radical surgical excision is the most common and most successful method for treatment of this lesion, but the utility of this approach is dependent 


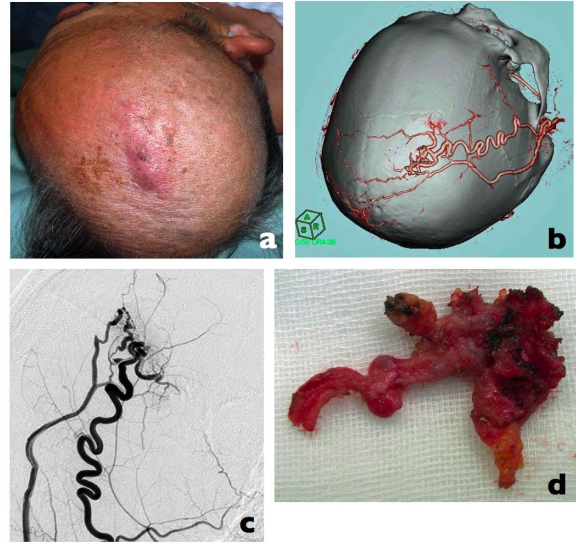

Figure 1. (a) The preoperative photograph demonstrates the extent of scalp arteriovenous malformation (AVM); (b) Three-dimentional CT angiography (3D-CTA) shows the dilated tortuous superficial temporal artery (STA) and the nidus and venous drainage into the superficial scalp vein; (c) Cerebral angiography revealed similar findings; (d) Resected surgical specimen.

on the size of the lesion, the number of feeders, the draining pattern, patient preference and the local availability of experienced surgeons. The AVM in the present case was relatively small [4] and was deemed appropriate for surgical resection. This approach resulted in an excellent outcome. While follow-up of this lesion can be easily and noninvasively performed via 3D-CTA, longer follow-up periods are necessary to analyze the efficacy of the treatment. Although DSA is the gold standard for diagnosis of this lesion, the present case suggests that 3D-CTA is also useful for diagnosis, treatment planning, and follow-up of small extracranial scalp AVMs.

\section{CONCLUSION}

Surgical excision resulted in an excellent outcome when used for the management of a small scalp AVM in this case. Unlike large scalp AVMs, small scalp AVMs can be diagnosed, managed and followed using 3D-CTA.

\section{REFERENCES}

[1] Mohamed, W.N., Abdullar, N.N. and Muda, A.S. (2008) Scalp arteriovenous malformation: A case report. Malaysian Journal of Medical Sciences, 15, 55-57.

[2] Hage, Z.A., Few, J.W., Surdell, D.L., et al. (2008) Modern endovascular and aesthetic surgery techniques to treat arteriovenous malformations of the scalp: Case illustration. Surgical Neurology, 70, 198-203. doi:10.1016/j.surneu.2007.04.016

[3] Hastruk, A.E., Erten, F. and Ayata, T. (2012) Giant nontraumatic arteriovenous malformation of the scalp. Asian Journal of Neurosurgery, 7, 39-41. doi:10.4103/1793-5482.95698

[4] Kumar, R., Sharma, G. and Sharma, B.S. (2012) Management of scalp arteriovenous malformation: Case series and review of the literature. British Journal of Neurosurgery, 26, 371-377. doi:10.3109/02688697.2012.654838

[5] Nagasaka, S., Fukushima, T., Goto, K., et al. (1996) Treatment of scalp arteriovenous malformation. Neurosurgery, 38, 671-677. doi:10.1227/00006123-199604000-00007

[6] Matsushige, T., Kiya, K., Satoh, H. et al. (2004) Arteriovenous malformation of the scalp: case report and review of the literature. Surgical Neurology, 62, 253-259. doi:10.1016/j.surneu.2003.09.033 